[35] 5. Der Zinsanspruch des Kl. ergibt sich aus $₫ 8$ Abs. 1 des Vertrages nach $\$ 120$ Abs. 2 SGB V (Grundvertrag) für das Land Bad.-Württ. Danach hat die KK die Rechnung innerhalb von 30 Tagen nach Rechnungsstellung zu bezahlen (S. 1); für die Verzugszinsberechnung verweist S. 2 auf die Landesregelung nach $\$ 112$ Abs. 2 Nr. 1 SGB V. \19 Abs. 3 dieser Landesregelung für die Vergütung stationärer Leistungen sieht als Zinssatz fünf Prozentpunkte über. dem Basiszinssatz vor.

[36] Diese vertragliche Vereinbarung ist hier maßgeblich. Das entspricht der Rspr. des 1. Senats des BSG zur Verzinsungspflicht der KKn bei fälligen Krankenhausrechnungen, soweit entsprechende Vereinbarungen bestehen (BSG, SozR 4-2500 \$ 69 Nr. 7; SozR 4-5562 \&11 Nr. 2). Dieser Grundsatz gilt auch, soweit von $\$ 120$ Abs. 2 SGB V erfasste, ambulante Leistungen auf der Basis von vertraglichen Vereinbarungen unmittelbar von den $\mathrm{KKn}$ zu vergüten sind. Der Senat hält allerdings daran fest, dass Nachzahlungen vertragsärztlicher Honorare grundsätzlich nicht zu verzinsen sind, auch soweit Krankenhäuser auf gesetzlicher Grundlage an der vertragsärztlichen Versorgung teilnehmen (BSG SozR 4-2500 \$43b Nr. 1, Rdnrn. 52-53). Eine solche Konstellation liegt hier nicht vor, weil die hier betroffenen Laborleistungen der Hochschulambulanzen im Rahmen des Screenings nicht von der KÄV aus der vertragsärztlichen Gesamtvergütung honoriert werden. [...]

https://doi.org/10.1007/s00350-018-5107-5

\section{Anmerkung zu BSG, Urt. v. 16.5.2018 - B 6 KA 45/16R (LSG Bad.-Württ.)}

\section{Kerrin Schillhorn}

Mit seiner Entscheidung vom 16.5.2018 hat sich der 6. Senat des BSG klar gegen den formalen Auslegungsansatz der Instanzgerichte ausgesprochen und den deutlich praxisnäheren Ansatz der landesübergreifenden Geltung von Verträgen nach $\$ 120$ Abs. 2 SGB V gewählt. Zutreffend hält der Senat gesonderte Verträge für jedes Bundesland nicht für notwendig und weist darauf hin, dass bei Anwendung des Ansatzes der Instanzgerichte jede Hochschulambulanz nicht nur Verträge mit den Krankenkassen des eigenen Bundeslandes treffen müsste, sondern auch mit allen Krankenkassen der anderen Bundesländer, um seine Leistungen für Patienten aus anderen Bundesländern vergütet $\mathrm{zu}$ erhalten. Da gerade Hochschulambulanzen jedoch Patienten über den regionalen Einzugsbereich hinaus behandelten, sei dies nicht zu verlangen. Unterstützt wird diese Regelung durch die Entscheidung des Gesetzgebers, im Jahre 2017 eine „klarstellende“ Ergänzung des \$120 Abs. 2 SGB dahin gehend vorzunehmen, dass, ,die Höhe der Vergütung für die Leistungen der jeweiligen Hochschulambulanz gilt auch für andere Krankenkassen im Inland, wenn deren Versicherte durch diese Hochschulambulanz behandelt werden".

Mit diesem Ansatz, aber auch der Entscheidung, nicht die vereinbarte Entgelthöhe unabhängig von der angemessenen Gegenleistung für die erbrachte Laborleistung zur Anwendung zu bringen, löst sich das BSG von der formalen Anforderung des Vertrages selbst. Es scheint, als sei das Urteil sowohl im Hinblick auf die Geltung des Vertrages über das einzelne Bundesland hinaus, als auch im Hinblick auf die vereinbarte Entgelthöhe mit dem Ziel eines praktikablen

Rechtsanwältin Dr. iur. Kerrin Schillhorn, MIL,

Fachanwältin für Verwaltungsrecht

und Fachanwältin für Medizinrecht,

michels.pmks Rechtsanwälte Partnerschaft mbB

Hohenstaufenring 57, 50674 Köln, Deutschland
Ergebnisses getroffen worden, nicht so sehr in Ansehung der rechtlich-formalen Herangehensweise. Letzteres mag durch die deutlichen Hinweise im Gesetzgebungsverfahren zur Ergänzung des $\$ 120$ Abs. 2 SGB V gut zu vertreten, wenn nicht gar zwingend sein. Wenn aber im ersten Schritt die Anwendbarkeit des Vertrages für die Hochschulambulanz und deren Vergütung für die Krankenkassen anderer Bundesländer bejaht wird, stellt sich die Frage, auf welche rechtliche Grundlage die „Anpassung“ der vertraglich vereinbarten Vergütung zu Lasten der Hochschulambulanz sich stützt. Inhaltlich und bezogen auf ein ,gerechtes“ Ergebnis ist die Entscheidung auch insoweit gut nachvollziehbar, rechtlich indes nicht ohne Weiteres.

Neben diesem wesentlichen Inhalt des Urteils enthalten die Entscheidungsgründe noch einige lesenswerte Erwägungen, wie z. B. die Feststellung, dass es sich bei Laborleistungen auf Überweisung von Belegärzten nicht um allgemeine Krankenhausleistungen handelt, und die Vergütung von Hochschulambulanzen nicht Gegenstand der vertragsärztlichen Versorgung und damit nicht von der Verzinsung des Anspruchs ausgenommen ist.

\section{Verlust des Rechts auf Wiederholung der Ausschreibung bei unberechtigter Einflussnahme des Praxisabgebers auf das Nachbesetzungsverfahren}

SGB V §103 Abs. 3a, Abs. 4; GG Art. 19 Abs. 4

1. Tatbestandliche Voraussetzung für die Nachfolgezulassung ist die Existenz einer fortführungsfähigen Praxis. Aus Gründen des effektiven Rechtsschutzes ist maßgeblicher Zeitpunkt für die Beurteilung der Fortführungsfähigkeit grundsätzlich der Zeitpunkt der Stellung des Antrags auf Ausschreibung.

2. Das Nachbesetzungsverfahren ist in der Regel mit der Rücknahme des Antrags auf Ausschreibung durch den Praxisabgeber beendet. Hat der Praxisabgeber den Antrag auf Ausschreibung seines Sitzes zurückgenommen, ist ein erneuter Antrag nur dann beachtlich, wenn der Arzt ein berechtigtes Interesse für die Rücknahme und die erneute Antragstellung darlegen und nachvollziehbar begründen kann.

3. Liegt ein solches berechtigtes Interesse nicht vor, verbleibt es bei dem Grundsatz, dass das Nachbesetzungsverfahren nach der Rücknahme des ersten Antrages beendet ist. Das Recht auf Nachbesetzung erlischt in diesem Fall. (Leitsätze der Bearbeiter)

BayLSG, Urt. v. 22.3.2017 - L 12 KA 77/16 ZVW (SG München)

Problemstellung: Das BSG (Urt. v. 23.3.2016 B 6 KA 9/15 R) hatte sich anlässlich der Klage eines zur vertragsärztlichen Versorgung zugelassenen Orthopäden, der nach bestandskräftiger Entziehung seiner Zulassung seine Praxis auf einen Nachfolger übertragen wollte, mit den tatbestandlichen Voraussetzungen der Nachfolgezulassung auseinanderzusetzen. Konkret ging es um die Frage, ob im maßgeblichen Beurteilungszeitpunkt eine fortführungsfähige Praxis vorlag. Besondere Brisanz erhielt der Fall dadurch, dass der Praxisabgeber seinen Antrag auf Ausschreibung seines Vertragsarztsitzes trotz vorhandener Bewerber zweimal zurücknahm

Eingesandt von Christian Pinnow, Berlin;

bearbeitet von Rechtsanwältin Dr. iur. Kristina Raske

und Rechtsanwalt Dr. iur. Christian Wittmann,

BRP Renaud \& Partner mbH

Königstraße 28, 70173 Stuttgart, Deutschland 